
approches théoriques et méthodologiques

\title{
Virtualité, représentation, signification : approche de la complexité documentaire
}

Virtuality, Representation, Signification: Approach to Documentary Complexity

\section{Caroline Courbières}

\section{(2) OpenEdition}

Journals

Édition électronique

URL : http://journals.openedition.org/edc/4100

DOI : $10.4000 /$ edc. 4100

ISSN : 2101-0366

Éditeur

Université Lille-3

Édition imprimée

Date de publication : 1 décembre 2012

Pagination : 103-116

ISBN : 978-2-917562-08-6

ISSN : $1270-6841$

Référence électronique

Caroline Courbières, « Virtualité, représentation, signification : approche de la complexité

documentaire », Études de communication [En ligne], 39 | 2012, mis en ligne le 01 décembre 2014, consulté le 02 mai 2019. URL : http://journals.openedition.org/edc/4100 ; DOI : 10.4000/edc.4100

Ce document a été généré automatiquement le 2 mai 2019.

(c) Tous droits réservés 


\title{
Virtualité, représentation, signification : approche de la complexité documentaire
}

Virtuality, Representation, Signification: Approach to Documentary Complexity

\author{
Caroline Courbières
}

\section{Introduction}

1 Le concept de document fonde la documentation en tant qu'ensemble de pratiques, dispositifs et usages. Il justifie, tout en dépendant d'elle, l'activité de tous les acteurs que l'on peut désigner sous l'appellation de "professionnels de l'information ». Pourtant, un document, ça n'existe pas... Cette entrée en matière n'est pas aussi provocatrice qu'elle semble l'être si l'on daigne se poser la question que lançait Jean Meyriat dans le cadre de sa réflexion sur le territoire des Sciences de l'information et de la communication : «ET LA SÉMIOLOGIE ?» (Meyriat, 1993a). Comment définir l'objet documentaire en faisant abstraction de la charge signifiante de son contenu informationnel ? Comment expliquer la possibilité d'une représentation documentaire déconnectée d'une éventuelle signification documentaire? Plus largement, comment envisager la documentation, discipline improbable au regard des Sciences de l'information et de la communication? Ces quelques interrogations jalonnent l'itinéraire que suit cet article entre virtualisation et actualisation.

\section{L'impossible définition du document}

2 L'évocation des étapes fondatrices de la réflexion sur le concept de document nous invite tout d'abord à confronter les notions de signe, d'information et de savoir - défini en tant que formes de connaissance reconnues par une société (Jeanneret, 2007)-, selon lesquelles se déploie sa dimension épistémologique. Dans les années trente, Paul Otlet 
propose une première définition de l'objet documentaire caractérisé par un support et un signe (Otlet, 1934, 13). À l'aube des années cinquante, Suzanne Briet reprend ces deux caractéristiques fondamentales qui identifient le document en tant qu'objet signifiant. Elle intègre dans sa propre typologie documentaire l'animal et l'objet du monde naturel tout en introduisant la notion d'unité documentaire comme objet de la quête scientifique (Briet, 1951, 9) ; ce faisant, elle pose tout à la fois la nature polysémiotique de l'objet documentaire et le nécessaire processus de transformation de l'objet en objet documentaire. Conservant la notion de support posée par Paul Otlet et la nature sémiotique de l'objet avancée par Suzanne Briet, Jean Meyriat introduit celle d'information pour définir ce qu'est un document : « un objet sur lequel sont enregistrés des signes représentant de l'information. Ces signes n'appartiennent pas nécessairement au langage écrit; et s'ils lui appartiennent, l'objet peut être fait de bien d'autres matières que du papier » (Meyriat, 1993b, 152).

3 En reliant à son tour la notion d'information (documentaire) à celle de signe, Jean Meyriat souligne la nuance fondamentale posée en son temps par Robert Escarpit entre la notion d'information et la notion de savoir (Escarpit, 1991, 203) - nuance qui doit être entendue à la lumière de la différence essentielle de nature entre l'information mathématique et l'information sociale largement explicitée par Yves Jeanneret (Jeanneret, 2007). La notion d'information évoquée par Robert Escarpit renvoie à l'information mathématique, la notion de savoir s'apparente à l'information sociale et son inscription documentaire ; si la première se saisit dans une logique de transmission linéaire, la seconde implique un travail interprétatif qui déborde le modèle traditionnel de la communication et prouve "que le savoir et le sens ne sont jamais simplement donnés, mais qu'ils sont élaborés » (Jeanneret, 2008, 38). L'information en tant que donnée accède au statut d'information en tant qu'élément de connaissance à partir du moment où elle est saisie comme signe.

La référence au signe comme caractéristique de l'information documentaire sous-tend de la même manière la définition autorisée de l'information dans le Vocabulaire de la documentation: "élément de connaissance susceptible d'être représenté, à l'aide de conventions pour être conservé, traité ou communiqué. Toute information se caractérise par un contenu (signifiant), un signifié et une forme» (Boulogne, INTD-ER, 2004). L'éventualité d'une représentation de l'information documentaire inscrite dans sa définition même préfigure la virtualité de l'objet qui ne devient documentaire qu'à partir du moment où il participe d'une stratégie informationnelle située. C'est ainsi que Robert Escarpit a défini le document comme un « anti-événement » :

$\mathrm{Au}$ cours des derniers millénaires, l'homme a élaboré pour échapper à cette contrainte [l'événement] un anti-événement qui est le document [...] le pas décisif a été franchi lorsque l'homme a institué le document, cumulation de traces fixes et permanentes [...] où les réponses données en feed-back, à travers le temps, aux expériences antérieures, restent disponibles pour une lecture, c'est-à-dire une exploration libre de toute contrainte événementielle ou chronologique, en fonction du projet et de la stratégie destinée à le réaliser (Escarpit, 1991, 203).

5 Bien que marquée par la terminologie héritée de la théorie mathématique de l'information-communication, cette citation de Robert Escarpit rejoint la distinction fameuse faite par Jean Meyriat entre document par intention (l'objet produit en vue d'informer) et document par attribution (l'objet élaboré dans la recherche d'information) (Meyriat, 2001a, 144).

6 Selon cette distinction, l'objet documentaire dépend bien d'une "stratégie destinée à le réaliser ». Cette stratégie évoquée, qui est double et asymétrique, situe l'objet 
documentaire à deux moments différents: le moment de son intentionnalité et le moment de son utilisation. À partir de ces deux visées communicationnelles distinctes, nous avons insisté sur la notion de virtualité comme fondatrice de l'objet documentaire, le moment de l'interprétation du document primant sur celui de sa production; tout objet se voulant document ne le sera qu'à partir du moment où il sera (re)construit en tant que tel, l'absence de (re)construction du sens d'un objet documentaire invalidant son statut même de document (Courbières, 2008). Un objet documentaire n'existe donc qu'à partir du moment où il signifie pour quelqu'un. Pointer l'action institutionnalisante du récepteur dans la définition de l'objet documentaire impose en même temps de l'appréhender à partir de son actualisation en tant qu'objet signifiant. L'on doit ainsi concevoir une sorte de pré-statut documentaire potentiel d'un objet pouvant être soumis à la description ou l'analyse, puisque, à l'image du signe, le document dépend de «ce moment instituant, celui du regard prêt à interpréter » que pose Yves Jeanneret à propos du support documentaire (Jeanneret, 2007, 100).

7 C'est suivant cette virtualisation/actualisation de l'objet documentaire que nous avons avancé le principe de l'horizon documentaire (Courbières, 2004 ; Courbières et Couzinet, 2006), en reprenant les concepts d'horizon d'attente développé en esthétique de la réception: l'horizon d'attente tel que le définit Hans Robert Jauss embrasse les phénomènes de réception pendant la production de l'objet artistique et après sa création ; il concerne à la fois le producteur de l'œuvre et ses récepteurs. L'objet implique un «horizon d'attente littéraire », c'est-à-dire «tout un ensemble d'attente et de règles du jeu avec lesquelles les textes antérieurs l'ont familiarisé et qui, au fil de la lecture peuvent être modulées, corrigées, modifiées ou tout simplement reproduites » (Jauss, 1978, 51), tout en impliquant un «horizon d'attente social », à savoir, « la disposition d'esprit ou le code esthétique des lecteurs, qui conditionne la réception » (Jauss, 1978, 259). L'attente en question présuppose l'activité du sujet récepteur et son appréhension de l'œuvre dans la médiation d'une intertextualité. L'horizon documentaire, à l'image de l'horizon d'attente, n'est pas figé, il se modifie et projette la com-préhension de l'objet documentaire dans un ensemble d'objets que le sujet récepteur (ré)active. Or le caractère variable de l'horizon documentaire remet en question la stabilité informationnelle de l'objet documentaire, non pas en raison de la forme ou du support de ce dernier, mais parce que le document se construit à partir de son contexte de réception. C'est sur cette (non) définition du document (pris comme un artefact) et sur l'horizon documentaire qui la subsume que la notion de représentation documentaire prend appui.

\section{La problématique de la représentation documentaire}

8 Cette problématique peut prendre place au centre du débat classique pour les théoriciens du langage entre la fonction représentationnelle et la fonction constructive (du langage). Nous avons eu l'occasion d'examiner la question de la représentation à travers l'analyse des langages documentaires. Ces outils professionnels, qui peuvent également être appréhendés en tant qu'objets documentaires constituent en effet un espace privilégié par leur double statut, à la fois outils de description des objets documentaires et discours construisant leurs propres représentations ; on a ainsi pu montrer comment ces langages, censés représenter des objets référentiels (de la connaissance), construisent leur propre contenu informationnel à travers lequel ils deviennent objets d'interprétation. La représentation documentaire réside alors dans cette tension entre un dit documentaire et 
un dire documentaire (Courbières, 2011). En d'autres termes, et à partir de la distinction posée en 1892 par Frege entre le sens (der Sinn) et la dénotation (die Bedeutung), on peut convenir que, d'une part, la saisie des langages documentaires en tant qu'outils vise à déterminer la dénotation des propositions documentaires, c'est-à-dire leur fonction de désignation des connaissances à représenter. D'autre part, ces langages, en tant que systèmes de signes, autorisent une seconde saisie qui s'intéresse à leur sens, résultant de la configuration signifiante constituée par des unités documentaires définies les unes par rapport aux autres. Si nous faisons référence aux travaux du philosophe et logicien Gottlob Frege en liaison avec la tension observée dans le fonctionnement même des langages documentaires, c'est parce qu'ils nous semblent trouver un écho contemporain dans la tension évoquée par Bernd Frohmann entre la matérialité du document et sa fonction représentationnelle (Frohmann, 2011). Néanmoins, il faut noter que la représentation documentaire ne renvoie pas à ce que Gottlob Frege désigne sous le terme de représentation - qui reste pour lui essentiellement individuelle (Frege, 1971, 105-106) -, mais bien au sens et à la dénotation tout à la fois.

9 Plus précisément, et en prolongeant cette approche philosophique, on peut affirmer que la représentation à l'œuvre dans les systèmes documentaires combine les différences de fonction qu'assument le langage et le langage technique : tandis que le langage va de la signification à la désignation, le langage technique opère le trajet inverse, de la désignation à la signification (Coseriu, 2001). L'objet documentaire lui-même se place à l'intersection de ces deux fonctions : un document répond à la fonction de désignation par les données qui constituent son expression, et c'est par l'actualisation de son contenu qu'il acquiert un sens, devenant un signe à part entière. Si l'on consulte la définition technique de l'objet documentaire, on constate que la notion de signe utilisée par les pères de la documentation se trouve pulvérisée à travers celles d'information (documentaire), d'informations (au sens de données), de la signification attribuée, et de la visée communicationnelle plurielle de l'objet : un document est «l'ensemble d'un support d'information, quel qu'il soit, des données enregistrées sur ce support et de leur signification, servant à la consultation, l'étude, la preuve ou la trace, etc.: livre, échantillon de parfum, tissus, film, etc. Le tout constitue une unité autonome" (Boulogne, INTD, 2004). On retrouve dans cette définition l'aspect indissociable de l'objet documentaire et de son pouvoir-signifier, à la manière dont Ferdinand de Saussure reliait le signe linguistique à la notion de signification: « Ce sont deux formes du même concept de l'esprit, vu que la signification n'existerait pas sans un signe, et qu'elle n'est que l'expérience à rebours du signe, comme on ne peut pas découper une feuille de papier sans entamer l'envers et l'endroit de ce papier, du même coup de ciseaux » (Saussure, 2002, 96). Si tout objet peut prétendre à un statut documentaire - à l'image de l'antilope prise comme exemple par Suzanne Briet ou de pièces vestimentaires présentées dans des expositions muséales -, c'est en vertu de la fonction informative qu'un récepteur est susceptible de lui attribuer.

La virtualité de l'objet documentaire et sa charge sémantique intrinsèque l'inscrivent explicitement au centre du mécanisme dynamique de la communication. Le linguiste Bernard Pottier a modélisé ce double processus par une représentation des parcours onomasiologique - qui rend compte des phénomènes de désignation (conceptualisation et sémiotisation) - et sémasiologique - qui rend compte des phénomènes d'interprétation (identification et compréhension). 


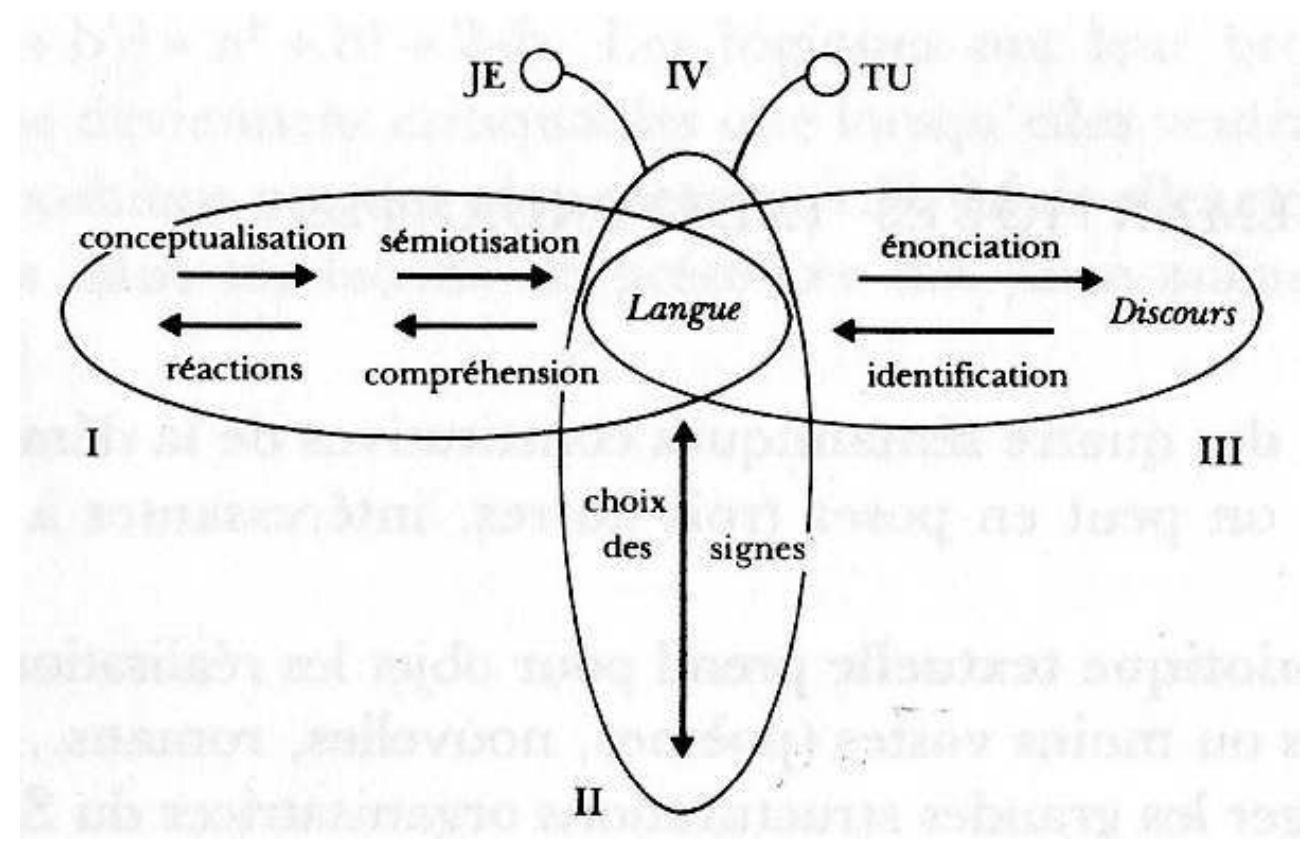

Figure 1 : le papillon sémantique de Bernard Pottier

11 À partir de l'articulation de ces deux parcours, Bernard Pottier distingue quatre lieux d'analyse possible d'appréhension du sens: le référentiel (externe) comme «monde de départ ou d'arrivée, facultatif », le conceptuel (ou référentiel interne) comme lieu de la représentation mentale, la langue comme savoir, et le discours, « dans sa double fonction de résultat observable [...] et de base de départ pour l'interprétant » (Pottier, 1992a, 18). Cette mise à plat des possibilités de production et d'interprétation du sens permet de dresser l'étendue d'une théorie générale qui peut aussi bien viser à comprendre le sens d'une expression sur la base de la structure sémantique - objet de la sémantique formelle d'inspiration logique dite vériconditionnelle-, que s'occuper des relations entre les niveaux référentiels et le système de la langue - tâche relevant de la sémantique référentielle-, ou encore s'attacher de manière privilégiée à l'analyse des formes signifiantes résultant de la mise en signe opérée.

Ces deux derniers angles d'attaque doivent ainsi être choisis pour déterminer la double nature des relations sémantiques qui structurent tout langage documentaire : « relations between concepts, senses or meanings should not be confused with relations between the terms, words, expressions or signs that are used to express the concepts. It is, however, common to mix both of these kinds of relations under the heading 'semantic relations' " (Hjørland, 2007). Concernant l'objet documentaire, c'est bien à sa matérialité signifiante que l'on doit s'attacher en priorité, à la fois comme manifestation d'une représentation et comme actualisation de connaissances, joignant dès lors le phénomène de la connotation au fait de dénotation: «la dénotation renvoie essentiellement à la conceptualisation du référent ; la connotation est dépendante du savoir (cognitif), du vouloir (intentionnel), de l'énonciation, et, éventuellement du contexte et de la situation " (Pottier, 1992b, 74). Le problème de la désignation abordé précédemment se trouve alors rejeté de l'analyse première puisque c'est la signification de l'objet lui-même qui prime, indépendamment de ce à quoi il est susceptible de renvoyer aux niveaux conceptuel ou référentiel: « un signe n'est pas pourvu de sens parce qu'il désigne un référent » (Rastier, 2009, 21). Cette problématique, qui s'appuie sur les apports de la sémantique linguistique et textuelle, 
nous invite à réfléchir plus largement aux soubassements sémiologiques qui sous-tendent le cadre général d'une théorie de la documentation.

\section{La documentation comme discipline : la question du sens au cœur des Sciences de l'information et de la communication}

Apparu à la fin du XIXe siècle pour signifier l'action de réunir des documents, le terme documentation est adopté au début du XXe siècle par l'Institut international de bibliographie fondé par Paul Otlet et qui deviendra en 1931 l'Institut international de documentation. Rapidement imposé, documentation devient dans les années 1930 «le mot-centre d'une activité de recherche et de traitement des documents, organisée à cette époque» (Rey, 1998, 1114). Ce terme exprime bien à la fois l'objet en question (le document - terme dérivé, faut-il le rappeler, du verbe latin docere) et la nécessaire action qu'il implique (explicite à travers la dérivation nominale produite par le suffixe -tion) - : la documentation, en tant que discipline, ne se résume pas à l'application de techniques documentaires, elle suppose une certaine posture qui prend appui sur l'élaboration même de son objet (l'objet documentaire). Si peu d'études hexagonales portent précisément sur cette «discipline scientifique qui fournit les connaissances fondamentales et appliquées sur lesquelles s'appuie la pratique » (Meyriat, 1993c, 151), la documentation doit pourtant affirmer sa place au sein des Sciences de l'information et de la communication, dont la spécificité même a amené ses spécialistes à lister, classer, organiser ses diverses composantes (Couzinet, 2008).

Une cartographie des Sciences de l'information et de la communication reste extrêmement mouvante ; c'est ainsi qu'à l'origine de ce champ, les recherches sur les médias ou en journalisme s'inscrivaient sur le volet information, tandis qu'elles peuvent apparaitre aujourd'hui, pour certaines, comme le fer de lance du volet communication. Exception culturelle aidant, la situation est différente à l'étranger où les termes information et communication ne sont pas couplés. De fait, la revendication possible d'une science de l'information s'explique par l'importation du modèle américain qui, à partir de la seconde moitié du XXe siècle, impose l'Information Science (Fondin, 2005). L'étude menée récemment sur la représentation des Sciences de l'information et de la communication à travers le langage documentaire RAMEAU témoigne du statut instable de ces Sciences de l'information dans l'ensemble de ce champ et constate la prédominance de «termes associés au domaine des sciences de l'information en général et aux métiers de la documentation en particulier » (Boutin et al., 2008). Si l'on ne saurait taire l'asservissement de la documentation aux techniques documentaires dans la représentation commune de cette discipline, celle-ci ne peut être arrachée au substrat théorique qui oriente sa pratique : la documentation, en tant que discipline scientifique, doit bénéficier des "acquis d'une science pour laquelle le sens est la valeur décisive " (Meyriat, 2001b : 261). L'étude de documents, de leur traitement informationnel et de leur circulation implique en effet d'étudier la construction du sens des systèmes et des processus documentaires. L'exigence sémiologique, dont la visée est de décrire les conditions de production et de saisie du sens nous semble devoir guider, de fait, les études actuelles sur l'organisation des connaissances. 
Selon Jean Meyriat, la sémiologie devait légitimement être considérée comme une des sciences de la communication, puisqu'elle «étudie les moyens symboliques par lesquels se réalise la communication" (Meyriat, 1993d, 334). Projetée comme "une science qui étudie la vie des signes au sein de la vie sociale» (Saussure, 1972, 33), la sémiologie possède plusieurs histoires, et se dit aussi sémiotique selon l'approche que l'on souhaite mettre en valeur: outre l'institutionnalisation du terme sémiotique lors de la fondation en 1969 à Paris de l'Association Internationale de Sémiotique (International Association for Semiotic Studies), sous l'égide notamment de Roman Jakobson, André Martinet, Roland Barthes, Algirdas Julien Greimas et Umberto Eco, il existe une vision oppositionnelle qui consiste à renvoyer la sémiologie du côté des systèmes de signes, la sémiotique s'occupant des processus de signification. Par ailleurs, la sémiotique peut encore principalement se référer à la semiotics du philosophe et logicien américain Charles Sanders Peirce, ou renvoyer aux travaux du Cercle de Paris fondé en 1967 par A. J. Greimas dans le prolongement de la linguistique structurale. La sémiologie (ou sémiotique) européenne tire son origine du geste fondateur du linguiste suisse Ferdinand de Saussure qui consista à déconnecter le signe linguistique de l'objet extra-linguistique auquel il réfère. La notion saussurienne de signifié - liée à celle de signifiant et distincte de celle de concept -, permet d'analyser la construction du sens à partir du seul contenu linguistique, le signe n'ayant de valeur que par les rapports oppositionnels qu'il entretient avec les autres signes du système. Figure emblématique de ce courant européen, Roland Barthes conçut la sémiologie comme une branche de la linguistique, inversant par là même la proposition saussurienne: «la langue est le domaine des articulations, et le sens est avant tout découpage » (Barthes, 1964, 114). Ce programme se retrouve de fait si l'on veut penser la documentation dont « la question essentielle [est] celle des possibilités et modalités de catégorisation de l'information et de sa représentation » (Meyriat, 1993c, 151).

Dans un article sur la science de l'information, Jean Meyriat évoquait ainsi la linguistique et la sémiologie comme sciences pourvoyeuses d'un "équipement méthodologique " (Meyriat, 2001b, 256), un peu à l'image de l'outillage sémiotique qui sert les études marketing (Boutaud, 2007). De son côté, et mettant en perspective l'importance des semiotic studies par rapport au nombre peu élevé des départements de sémiotique de par le monde, le danois Niels Winfeld Lund souligne également l'apport de la théorie sémiologique à nombre de disciplines scientifiques (Lund, 2010). Certains travaux de linguistique et sémiotique peuvent d'ailleurs relever de la compétence des Sciences de l'information et de la communication «si l'objet de l'étude porte sur le fonctionnement d'un processus de communication, ou sur l'extraction et la représentation des connaissances en vue de la conception de systèmes d'information » (Miège, Odin, 1993, 8). La généalogie même des Sciences de l'information et de la communication rappelle enfin les liens privilégiés que celles-ci entretiennent avec le courant sémiologique ; en effet,

c'est à la rencontre [...] de personnalités représentant des courants scientifiques différents (A.G. Greimas et R. Barthes, pour la sémiologie et la sémiotique, Jean Meyriat pour les Sciences de l'information et de la documentation, Robert Escarpit, pour les sciences de la communication...), que tient l'idée d'un projet de constitution d'interdiscipline regroupant ces courants (Ollivier, 2001, 340-341).

17 Si la documentation en tant que discipline se situe précisément au cœur de cette interdiscipline, l'action documentaire, à la charnière d'un monde à représenter et d'un monde signifié, doit être saisie dans cette articulation du sens puisque son rôle est de 
permettre et faciliter l'accès aux connaissances à partir de l'organisation et de la représentation d'objets informationnels.

\section{Conclusion} rivée à ses aspects techniques, ne peut faire l'économie des principes de l'étude du sens et des significations. L'appréhension de l'information en tant que signe disponible pour la constitution d'une mémoire documentaire rejoint la théorisation du document dont la virtualité consubstantielle achève de proclamer son impossible définition. Si la voie sémiologique continue d'être empruntée comme cadre général d'une théorie de la documentation (Mai, 2001; Raber et Budd, 2003; Lund, 2010), c'est bien parce que les objets, systèmes ou processus documentaires ne peuvent être envisagés hors de cette «nébuleuse du sens » qu'évoquait Saussure.

\section{BIBLIOGRAPHIE}

Barthes R., (1964), « Éléments de sémiologie », Communications, 4, pp. 91-135.

Boulogne A. et INTD-ER, (2004), Vocabulaire de la documentation, Patis, ADBS Éditions, http:// www.adbs.fr/vocabulaire-de-la-documentation-41820.

Boutaud J.-J., (2007), « Du sens, des sens : Sémiotique, marketing et communication en terrain sensible », Semen, http://semen.revues.org : (consulté le 24/02/2012).

Boutin E., Liu P., Goria S., Dumas P. et Amos D., (2008), « Les SIC à partir du thésaurus Rameau : représentation ou interprétation? ", in Les sciences de l'information et de la communication: affirmation et pluralité, 16e Congrès de la SFSIC, 11-13 juin 2008, Compiègne, http://www.sfsic.org/ congres_2008/spip.php?article125.

Briet S., (1951), Qu'est-ce que la documentation?, Paris, EDIT.

Coseriu E., (2001), « Dix thèses à propos de l'essence du langage et du signifié », Texto !, vol. VI, n $\circ 2$, http://www.revue-texto.net/Inedits/Coseriu_Theses.html.

Courbières C., (2011), «Stéréotypage et artifice documentaires », Communication \& langages, n -170, pp. 97-106.

Courbières C., (2008), « La question documentaire à l'épreuve du numérique : le recours aux fondamentaux », Sciences de la Société, nº 75, pp. 40-51.

Courbières C., (2004), « Documents, signes et savoirs : retour sur l'analyse documentaire », in Metzger J.-P. (dir.), Médiation et représentation des savoirs, Paris, L’Harmattan, pp. 159-170.

Courbières C. et Couzinet V., (2006), « Du bleu horizon à l'horizon documentaire : représentation des connaissances à l'aube de la construction européenne », in Timimi I. et Kovacs S. (dir.), Indice, index, indexation: Actes du colloque international organisé les 3 et 4 novembre 2005 à l'Université Lille 3, Paris, ADBS, pp. 81-91.

Études de communication, 39 | 2012 
Couzinet V., (2008), « Représenter, répertorier, transmettre : formes d'institutionnalisation d'une discipline ", in Marteleto R. M. et Thiesen I. (dirs.), Médiations et usages des savoirs et de l'information : un dialogue France-Brésil, actes du 1er colloque du réseau MUSSI, Rio de Janeiro, 4-7 novembre 2008, pp. 63-81.

Escarpit R., (1991), L'information et la communication : théorie générale, Paris, Hachette.

Fondin H., (2005), « La science de l'information ou le poids de l'histoire », Les enjeux de la communication, http://w3.u-grenoble3.fr/les_enjeux/2005/Fondin/home.html.

Frege F. L. G., (1971), Écrits logiques et philosophiques, Paris, Points Seuil.

Frohmann B., (2011), « Reference, representation, and the materiality of documents », in Couzinet V. et Courbières C. (dirs.) Médiations et hybridations : construction sociale des savoirs et de l'information, actes du $2^{\text {e colloque scientifique international du réseau MUSSI, } 15-17 \text { juin 2011, Toulouse, Manifestations scientifiques }}$ du Réseau MUSSI, pp. 55-67.

Jauss H. R., (1978), Pour une esthétique de la réception, Paris, Gallimard.

Hjorland B., (2007), « Semantics and Knowledge Organization », Annual Review of Information Science and Technology (ARIST), vol. 41, pp. 367-405, http://dlist.sir.arizona.edu/2312.

Jeanneret Y., (2007), Y-a-t-il vraiment des technologies de l'information?, 2e édition, Villeneuve d'Ascq, Presses Universitaires du Septentrion.

Jeanneret Y., (2008), « La relation entre médiation et usage dans les recherches en informationcommunication ", in Marteleto R. M. et Thiesen I. (dirs.), Médiations et usages des savoirs et de l'information : un dialogue France-Brésil, actes du 1er colloque du réseau MUSSI, 4-7 novembre 2008, Rio de Janeiro, pp. 36-59.

Lund N. W., (2010), « Document, text and medium : concepts, theories and disciplines », Journal of Documentation, vol. 66, $n^{\circ}$ 5, pp. 734-749.

Mai J. E., (2001), « Semiotics and indexing : an analysis of the subject indexing process », Journal of Documentation, vol. 57, $\mathrm{n}^{\circ}$ 5, pp. 591-622.

Meyriat J., (1993a), « Langages documentaires », in Estivals R. (dir.), Les sciences de l'écrit : Encyclopédie internationale de bibliologie, Paris, Retz, pp. 351-352.

Meyriat J., (1993b), « Documents photographiques et électroniques », in Estivals R. (dir), Les sciences de l'écrit : Encyclopédie internationale de bibliologie, pp. 152-154.

Meyriat J., (1993c), « Documentation », in Estivals R. (dir.), Les sciences de l'écrit : Encyclopédie internationale de bibliologie, Paris, Retz, pp. 150-151.

Meyriat J., (1993d), « La bibliologie parmi les sciences de l'information et de la communication », in Estivals R. (dir.), Les sciences de l'écrit : Encyclopédie internationale de bibliologie, Paris, Retz, pp. 326-335.

Meyriat J., (2001a), « Document, documentation, documentologie », in Couzinet V. (dir), Jean Meyriat, théoricien et praticien de l'Information Documentation, Paris, ADBS Éditions, pp. 143-159.

Meyriat J., (2001b), « La science de l'information », in Couzinet V. (dir.), Jean Meyriat, théoricien et praticien de l'Information Documentation, Paris, ADBS Éditions, pp. 249-265.

Miège B. et Odin R., (1993), « Les domaines de compétence de la 71e section », La Lettre d'Inforcom , n 42, pp. 7-8.

Ollivier B., (2001), « Enjeux de l'interdiscipline », L'année sociologique, vol. 51, pp. 337-354. 
Otlet P., (1934), Traité de documentation : le livre sur le livre, Brussels, Éditions Mundaneum, Centre de Lecture Publique de la Communauté Française.

Pottier B., (1992a), Sémantique générale, Paris, PUF.

Pottier B., (1992b), Théorie et analyse en linguistique, 2e éd., Paris, Hachette.

Raber D. et Budd J. M., (2003), « Information as sign : semiotics and information sciences », Journal of Documentation, vol. 59, $\mathrm{n}^{\circ} 5$, pp. 507-522.

Rastier F., (2009), Sémantique interprétative, 3e éd., Paris, PUF.

Rey A. (dir.), (1998), Dictionnaire historique de la langue française, Paris, Dictionnaire Le Robert.

Saussure F. de, (1972), Cours de linguistique générale, Paris, Payot.

Saussure F. de, (2002), Écrits de linguistique générale, établis et édités par Simon Bouquet et Rudolph Engler avec la collaboration d'Antoinette Weil, Paris, Gallimard.

\section{RÉSUMÉS}

Le concept de document fonde la documentation en tant que praxis et discipline. L'itinéraire proposé dans cet article invite à considérer cet objet au regard des notions de signe, d'information et de savoir, selon lesquelles se déploie sa dimension épistémologique. Une tentative de définition approfondit ainsi la signification et la virtualité documentaires comme conditions préalables à la question de la représentation documentaire. Cette représentation se trouve mise en abyme au travers des systèmes d'organisation des connaissances que constituent les langages documentaires. Elle nous conduit in fine à réaffirmer la voie sémiologique comme cadre général d'une théorie de la documentation.

The concept of document is foundational within documentation as a praxis and a discipline. The goal of this paper is to consider the document with regard to the notions of sign, information, and knowledge through which its epistemological dimension is established. After proposing a definition, we posit the notions of documentary signification and virtuality as prerequisites for representation, which occurs especially in documentary languages. An approach based on semiology is thus considered a useful framework for the development of a theory of documentation.

\section{INDEX}

Mots-clés : document, information, théorie de la documentation, sciences de l' information et de la communication, sémiologie, sémiotique

Keywords : document, information, documentation studies, information sciences, communication sciences, semiology, semiotics

\section{AUTEUR}

\section{CAROLINE COURBIÈRES}

Caroline Courbières, LERASS - Université de Toulouse 